\title{
Reduction of Patella-baja and Pseudo-patella-baja Does Not Improve Range of Motion in Patients After Mega-TKA
}

\author{
TILMAN GRAULICH ${ }^{1}$, CAROLINE KRANZ $^{1}$, DAFANG ZHANG ${ }^{2,3}$, MARCUS OERGEL $^{1}$, TAREK OMAR PACHA ${ }^{1}$, \\ MARCO HAERTLE ${ }^{1}$, MOHAMED OMAR ${ }^{1}$, CHRISTIAN KRETTEK ${ }^{1}$ and MARTIN PANZICA ${ }^{1}$ \\ ${ }^{1}$ Trauma Department, Hannover Medical School, Hannover, Germany; \\ ${ }^{2}$ Department of Orthopaedic Surgery, Harvard Medical School, Boston, MA, U.S.A.; \\ ${ }^{3}$ Brigham and Women's Hospital, Boston, MA, U.S.A.
}

\begin{abstract}
Background/Aim: Patella baja $(P B)$ and pseudopatella baja (PPB) have been shown to negatively influence outcomes after total knee arthroplasty. We hypothesized that there is a high incidence of $P B$ and $P P B$ after megaprosthetic total knee arthroplasty (M-TKA), and that this is associated with reduced range of motion. Patients and Methods: We retrospectively analysed all patients in our Orthopaedic Trauma Department after distal femur or proximal tibia replacement. Preoperative and one-year postoperative followup included measurement of range of motion and detection of $P B$ and $P P B$ using radiological indices. Results: We included 44 patients (age: $73 \pm 19$ years). Preoperative $P B$ detected by ISI could be reduced from $13(36 \%)$ to $11(25 \%)(p<0.01)$. Preoperative vs. postoperative ISI was $0.88 \pm 0.23 \mathrm{vs}$. $1.06 \pm 0.45(p=0.03)$. $P P B$ was observed preoperatively in 23 (63\%) patients vs. 24 (54\%) postoperatively. Preoperative vs. postoperative CDI was $0.70 \pm 0.24$ vs. $0.95 \pm 0.43$ ( $p=0.002$ ). Preoperative flexion was $91^{\circ} \pm 30^{\circ}$ vs. $85^{\circ} \pm 24^{\circ}$ postoperatively ( $p>0.05)$. Conclusion: Both $P B$ and $P P B$ are frequently observed after M-TKA. A reduction in $P B$ and $P P B$ alone does not improve postoperative range of motion.
\end{abstract}

Physiological patellofemoral joint movement is important for normal knee function, i.e., flexion, extension, and force transmission. Patella baja (PB) is a real shortening of the patellar tendon, and pseudo-patella baja (PPB) is a relative shortening of the patellar tendon by elevation of the tibial plateau. $\mathrm{PB}$ and PPB

This article is freely accessible online.

Correspondence to: Tilman Graulich, MD, Trauma Department, Hannover Medical School, Carl-Neuberg-Straße 1, 30625 Hannover, Germany. Tel: +49 17615326723, Fax: +49 5115325877, e-mail: graulich.tilman@mh-hannover.de

Key Words: Revision total knee arthroplasty, patella baja, pseudopatella baja, outcome. are known to affect patellofemoral joint movement; a shortening of more than $10 \%$ of the patellar tendon is assumed to significantly reduce knee flexion due to the reduced length of the extensor apparatus (1-3). The consequences of PB and PPB are muscular imbalance and mechanical retropatellar overload, both of which can cause patellofemoral pain syndrome $(3,4)$. In $\mathrm{PB}$, the patellar tendon is at risk for rupture due to higher contact forces and an impingement-like situation of the patella against the tibial component (5). A relevant joint line elevation of more than $5-8 \mathrm{~mm}$ is believed to be clinically relevant and cause midflexion instability $(6,7)$.

Landmarks for joint line restoration in total knee arthroplasty (TKA) include the femoral epicondyles, the adductor tubercle, the fibular head, the tibial tubercle, and the inferior patellar pole (7). PB and PPB can be defined by measuring the InsallSalvati index (ISI), the modified Insall-Salvati index (mISI), the Caton-Dechamps index (CDI), and the Blackburne-Peel index (BPI) on lateral knee x-rays in $45^{\circ}$ knee flexion $(3,8,9)$. The postoperative incidence of $\mathrm{PB}$ ranges from $0.7 \%$ to $37 \%$, depending on the operative method $(2,5,10,11)$. PPB can be found in $25 \%$ to $47 \%$ of patients after primary TKA $(2,12)$. Behrend et al. have shown that PPB predicts impaired postoperative outcome with reduced range of motion after primary TKA. Chiang et al. have observed $58 \%$ and $82 \%$ incidence of PB and PPB, respectively, after revision TKA.

The incidence of $\mathrm{PB}$ and $\mathrm{PPB}$ and their relationship to clinical function after implantation of a distal femur or proximal tibia replacement remains unclear. The aim of this study was to evaluate incidence of PB and PPD and their relationship to function after megaprosthetic total knee arthroplasty (M-TKA).

\section{Patients and Methods}

We retrospectively analyzed all patients in our orthopaedic trauma department who underwent a MUTARS ${ }^{\circledR}$ distal femur or proximal tibia replacement (Implantcast $\mathrm{GmbH}$, Buxtehude, Germany) between 1998 and 2017. At the one-year follow-up (1y-fup) all patients who had lateral knee $\mathrm{x}$-rays and documented range of motion (ROM) were 

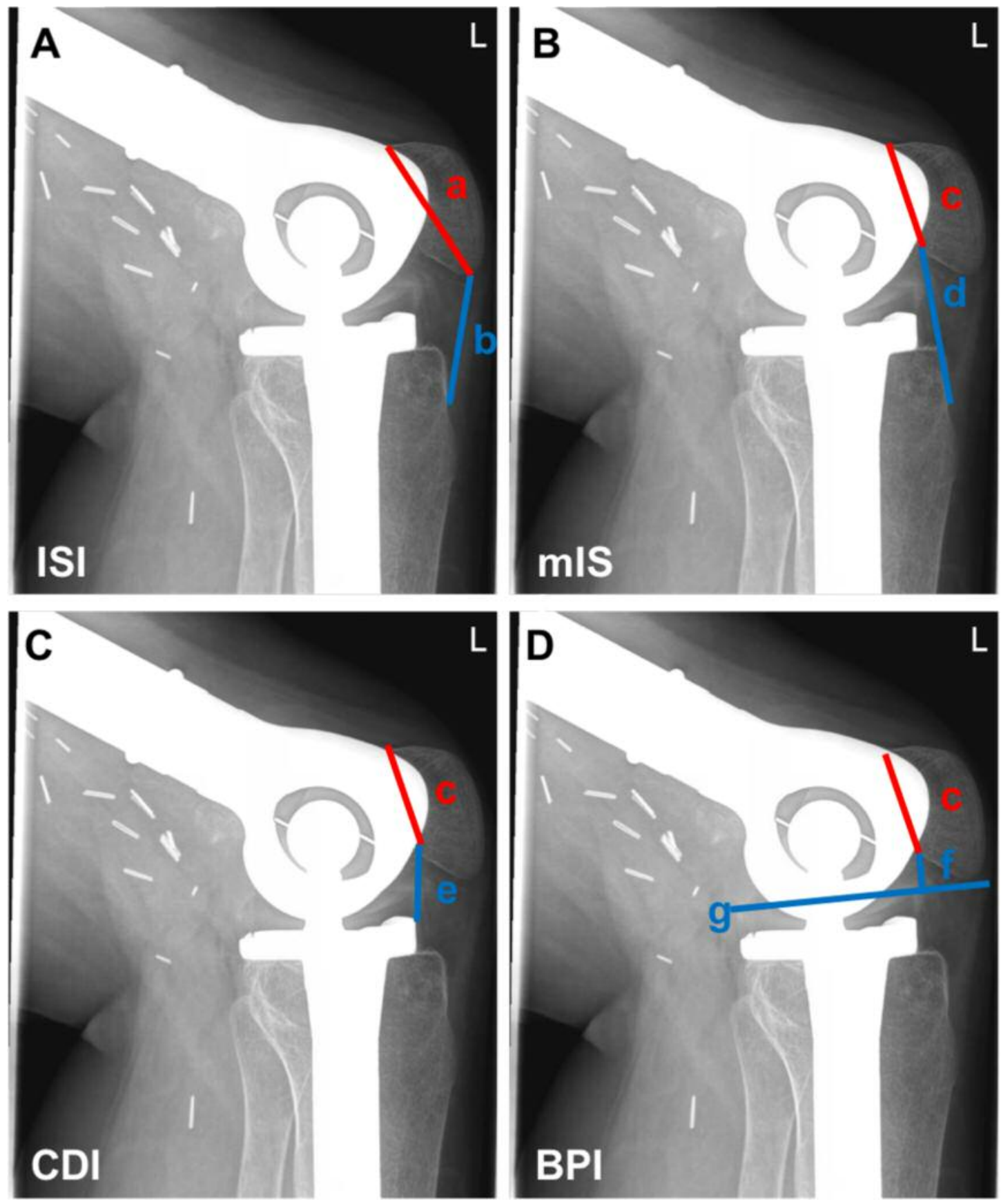

Figure 1. Lateral knee $x$-ray of a 25-year-old female patient 2 years after distal femur replacement. (A) ISI: Insall-Salvati index: dividing the length of the b) patella tendon by a line a) from the superior aspect to the inferior aspect of the patella (patella baja: $<0.8)(B) \mathrm{mISI}$ : modified InsallSalvati index: dividing the length of the d) patella tendon by the length c) of the patellar articular surface (patella baja: $<1.2)(C)$ CDI: CatonDechamps index: dividing the distance e) between the inferior pole of the patella and the upper limit of the tibial prothesis by the length c) of the patellar articular surface (pseudo-patella baja: <0.6), (D) BPI: Blackburne-Peel index: dividing the length $f$ ) of a perpendicular line g) from the inferior pole of the patella to the joint line by the length $c$ ) of the patellar articular surface (pseudo-patella baja: $<0.54$ ).

included. ROM was measured by a trained orthopaedic surgeon with a goniometer. Preoperative and postoperative lateral knee $\mathrm{x}$-rays were analyzed by a trained, independent orthopaedic surgeon with the PACS (Centricity Enterprise Web version 3.0, GE Medical Systems,
Milwaukee, WI, USA). An exact lateral radiograph was defined by the projection of both posterior femoral condyles over each other.

Radiological measurements included for detection of PB were the Insall-Salvati Index (ISI) and the modified Insall-Salvati Index 
Table I. PB and PPB indices as measured on lateral knee $x$-rays.

\begin{tabular}{|c|c|c|c|c|c|c|c|c|}
\hline & \multicolumn{4}{|c|}{ Preoperative } & \multicolumn{4}{|c|}{ Postoperative } \\
\hline & ISI & mISI & CDI & BPI & ISI & mISI & CDI & BPI \\
\hline Total & $0.88 \pm 0.23$ & $1.30 \pm 0.37$ & $0.71 \pm 0.24$ & $0.47 \pm 0.22$ & $1.06 \pm 0.45$ & $1.49 \pm 0.51$ & $0.95 \pm 0.43$ & $0.57 \pm 0.39$ \\
\hline $\mathrm{PB}$ & $0.64 \pm 0.12$ & $0.85 \pm 0.23$ & $0.47 \pm 0.19$ & $0.30 \pm 0.14$ & $0.85 \pm 0.26$ & $0.56 \pm 0.16$ & $0.48 \pm 0.19$ & $0.2 \pm 0.23$ \\
\hline PPB & $0.82 \pm 0.23$ & $1.15 \pm 0.37$ & $0.58 \pm 0.28$ & $0.34 \pm 0.12$ & $1.25 \pm 0.44$ & $0.82 \pm 0.32$ & $0.78 \pm 0.41$ & $0.29 \pm 0.2$ \\
\hline
\end{tabular}

PB: Patella baja; PPB: pseudo-patella baja; ISI: Insall-Salvati index; mISI: modified Insall-Salvati index; CDI: Caton-Dechamps index; BPI: Blackburne-Peel index.

(mISI). For detection of PPB, we measured the Blackburne-Peel Index (BPI) and the Caton-Dechamps Index (CDI) (Figure 1). The presence of $\mathrm{PB}$ was defined as $\mathrm{mISI}<1.2(9,10,12,13)$ and the presence of PPB as BPI $<0.54$ (14).

At least verbal informed consent was obtained from all individual participants who were included in the study. The study was approved by the local ethics committee (Nr 7957_BO_S_2018).

Statistical analysis. As the trial was of exploratory nature, no sample size calculation was performed. Data were summarized using Microsoft Excel ${ }^{\circledR}$ software and statistical analysis was performed with IBM SPSS Statistics ${ }^{\circledR}$ Version 25. Data were tested for normal distribution with the Kolmogorov Smirnoff Test. The $t$-test was used for comparative statistics for parametric data. The Mann-Whitney $U$-test was used for comparative statistics for nonparametric data. Correlation testing was performed using Pearson correlation for parametric variables and Spearman correlation for non-parametric variables. Data is presented as mean \pm standard deviation. Significance was defined at $p=0.05$.

\section{Results}

Demographic data. We included 44 patients with a mean age of $73 \pm 19$ years. Out of these, $28(63 \%)$ were female and 16 $(37 \%)$ were male. Mean body mass index (BMI) was $26 \pm 4$. Indication for M-TKA was periprosthetic fracture $(n=14)$, tumour $(n=12)$, infection $(n=8)$, traumatic fracture $(n=6)$, aseptic loosening $(n=2)$, and pathologic fracture $(n=2)$. MTKA was performed as the primary surgery in 20 patients and as revision surgery in 24 patients.

Radiological measurements. Preoperatively lateral knee x-rays were available in 36 patients and postoperatively in 44 patients. In 8 patients, preoperative lateral $\mathrm{x}$-rays were missing or not assessable due to trauma. PB could be observed by ISI in $13(36 \%)$ patients poreoperatively vs. $11(25 \%)$ patients postoperatively $(p<0.01)$, by mISI in $10(28 \%)$ patients preoperatively vs. $12(27 \%)$ patients postoperatively, and by CDI in $10(28 \%)$ patients preoperatively vs. 11 (25\%) postoperatively. The mean preoperative and postoperative ISI was $0.88 \pm 0.23 v s .1 .06 \pm 0.45(p=0.03)$, and the mean mISI was $1.30 \pm 0.37 v s .1 .49 \pm 0.51(p=0.06)$. PPB was observed in 23

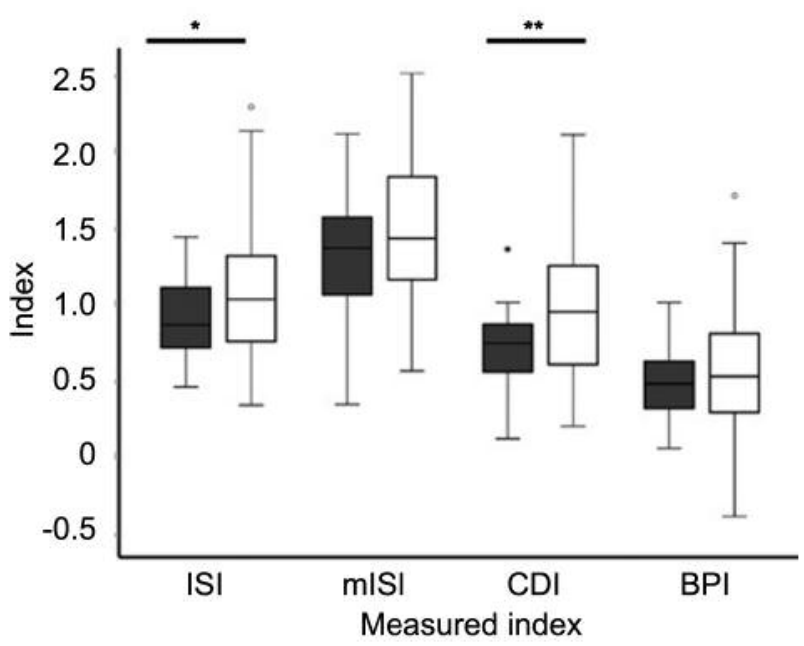

Figure 2. Preoperative and postoperative $P B$ and PPB indices. Grey: preoperative indices; white: postoperative indices.

(63\%) patients preoperatively vs. $24(54 \%)$ postoperatively. The mean CDI was $0.70 \pm 0.24$ preoperatively $v s .0 .95 \pm 0.43$ postoperatively $(p=0.002)$ and the mean BPI was $0.47 \pm 0.22$ preoperatively vs. $0.57 \pm 0.39$ postoperatively $(p=0.177$ ) (Table I, Figure 2).

In cases of distal femur replacement only, significant differences in CDI preoperatively and postoperatively were found $(0.64 \pm 0.21$ vs. $0.85 \pm 0.34 ; p<0.01)$. In cases of proximal tibia replacement only, ISI $(1.09 \pm 0.20 \mathrm{vs}$. $1.63 \pm 0.13 ; p<0.01)$ and mISI $(1.68 \pm 0.14 v s .2 .17 \pm 0.14$; $p<0.01)$ showed significant higher values postoperatively. In cases of combined distal femur replacement and proximal tibia replacement, no differences between preoperative and postoperative values in ISI, mISI, CDI and BPI were observed $(p>0.05)$. Postoperatively, distal femur replacement showed significant higher ISI, mISI, CDI and BPI than proximal tibia replacement (each: $p<0.01$ ), but not in cases with combined distal femur and proximal tibia replacements (Figure 3). 


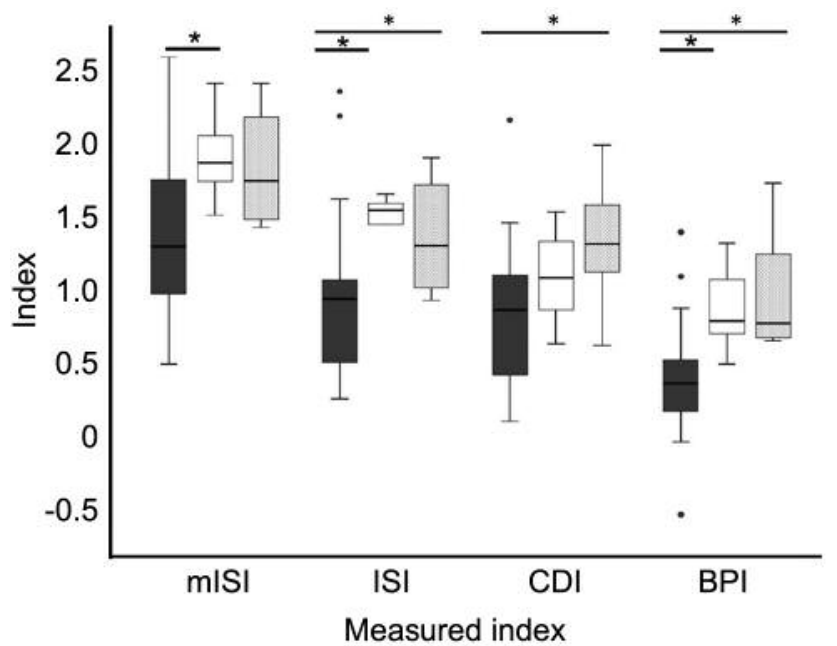

Figure 3. Postoperative indices in distal femur replacement, proximal tibia replacement, and both. Grey: Distal femur replacement ( $n=33)$, white: proximal tibia replacement $(n=5)$, dots: both $(n=6) . *$ Significant $p<0.05$.

Revision surgery. Whereas a reduced CDI could be observed preoperatively in cases of revision surgery compared to primary surgery $(0.64 \pm 0.22 v s .0 .81 \pm 0.26 ; p=0.037)$, no differences between primary surgery and revision surgery could be observed postoperatively for ISI, mISI, CDI, and BPI $(p>0.05)$ (Figure 4). No differences between preoperative and postoperative ISI, mISI, CDI and BPI were observed in cases of primary surgery $(p>0.05)$. CDI showed higher values postoperatively than preoperatively in cases of revision surgery (0.64 \pm 0.21 vs. $0.90 \pm 0.39 ; p<0.01)$. ISI, mISI, and BPI tended to be higher postoperatively $(p<0.1)$. We could not show any influence of age or BMI on the incidence of PB or PPB.

Range of motion. Preoperative range of motion was obtained in non-traumatic patients, except 22 who were not able to participate due to pain. Knee flexion was $91^{\circ} \pm 30^{\circ}$ preoperatively vs. $85^{\circ} \pm 24^{\circ}$ postoperatively $(p>0.05)$. Extension gap was $5^{\circ} \pm 9^{\circ}$ preoperatively $v s .4^{\circ} \pm 8^{\circ}$ postoperatively $(p>0.05)$. Postoperatively, we did not observe any significant reduction in the extension gap $\left(5^{\circ} v s .4^{\circ} ; p=0.32\right)$ or knee flexion $\left(80^{\circ} v s .86^{\circ}\right.$; $p=0.25$ ) in patients with $\mathrm{PB}$ compared to those without $\mathrm{PB}$. Patients with PPB showed a tendency for a larger extension gap compared to those without PPB $\left(5^{\circ}\right.$ vs. $\left.2^{\circ} ; p=0.16\right)$. Flexion tended to be reduced in patients with PPB compared to those without PPB $\left(81^{\circ} v s .89^{\circ}, p=0.12\right)$. Poor correlations between ISI, mISI, CDI, and BPI and range of motion were observed.

\section{Discussion}

In this study, we have described the incidence of PB and PPB in patients with megaprosthetic knee arthroplasty,

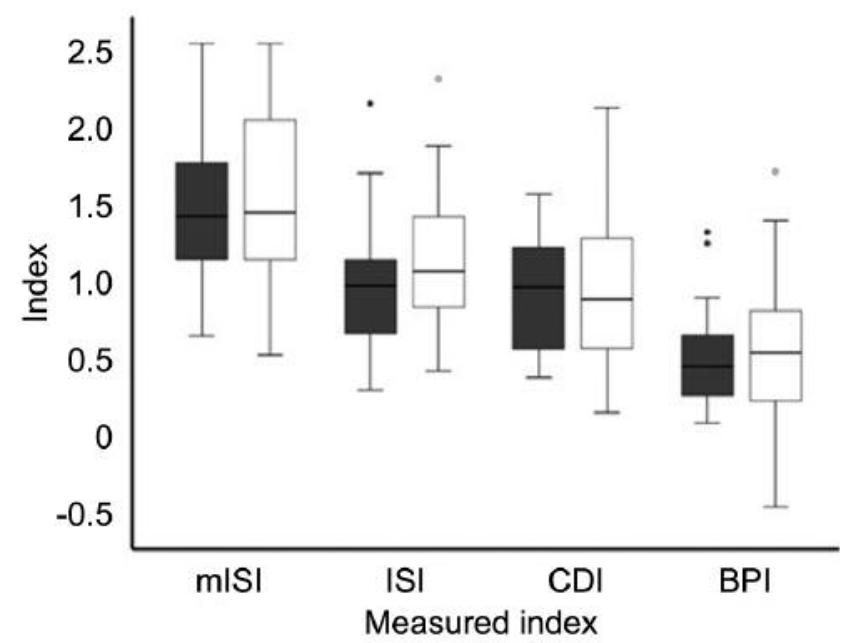

Figure 4. Postoperative indices of primary and revision surgery. Grey: primary surgery; white: revision surgery.

including distal femur and proximal tibia replacements. We showed that at $1 \mathrm{y}$-fup, $\mathrm{PB}$ could be reduced postoperatively from $36 \%$ to $25 \%$ and PPB from $63 \%$ to $54 \%$. These results are comparable to those in the literature after primary TKA for PB (0.7-37\%) and for PPB (25-47\%) $(2,12)$. However, while $\mathrm{PB}$ and $\mathrm{PPB}$ have been associated with a poorer outcome in primary TKA, we did not observe differneces in range of motion after M-TKA $(2,5,10,11)$. This was in accordance with Chiang et al., who also did not find any association between $\mathrm{PB}$ and $\mathrm{PPB}$ and decreased range of motion, which might be explained by preexisting reduced ROM preoperatively (3).

During revision surgery, preoperative $\mathrm{PB}$ and real shortening of the patellar tendon can be substantially reduced by intraoperative arthrolysis; consequently, we did not observe differences in postoperative patellar tendon length compared with patients undergoing primary surgery. Furthermore, no difference in ROM could be observed between patients with $\mathrm{PB}$ and patients without PB, suggesting that a minor shortening of the patellar tendon can be tolerated $(1,6,15-17)$. Shortening of the patellar tendon by more than $10 \%$ is expected to significantly reduce the knee flexion. With this assumption, we expected an improved range of motion in patients undergoing revision surgery with a postoperatively lengthened patellar tendon and reduced PB (1-3, 18). However, we did not observe improved flexion or reduced extension gap postoperatively. When comparing postoperative PPB to preoperative measurements, we observed an improvement in CDI but did not observe an improvement in patella height measured by BPI. Patients with postoperative PPB tended to show 
reduced flexion compared to patients without PPB. Therefore, we believe that PPB and exact joint line reconstruction are at least of equal importance for improvement of postoperative ROM.

The clinical relevance of joint line elevation is controversial. Generally, it is believed that a relevant joint line elevation of more than $4 \mathrm{~mm}$ is associated with retropatellar pain due to increased contact forces (relative overstuffing) and altered quadriceps mechanics (2, 10, 19). By contrast, minor joint line elevations do not seem to influence the postoperative clinical outcome after primary TKA $(2,4,6,13,17,20)$.

Whereas stability in extension and flexion by different extension and flexion gap sizes is a critical point in joint line reconstruction in primary TKA, in megaprosthetic TKA, this difficulty is avoided by a constrained endoprothesis design. More importantly, in M-TKA, joint line referencing and reconstruction can be achieved by preoperative planning with length measuring of the resected bone to determine the size of the modular endoprosthesis. Additive reconstruction of bone loss can be achieved by bone grafting or metal augmentations $(15,21)$. Larger defects require a modular revision endoprothesis such as the MUTARS system used here. Landmarks for joint line referencing are the femoral epicondyles, the adductor tubercle, the fibular head, the tibial tubercle, and the inferior patella pole $(7,22,23)$. If none of the mentioned landmarks are available, such as in severe traumatic injuries like floating knee injuries, referencing of the contralateral side could help to restore the joint line. Exact joint line reconstruction seems to be an important factor in postoperative ROM.

\section{Limitations}

Major limitations of this study are firstly the small and heterogeneous study cohort and the retrospective followup. Secondly, although we could observe a PPB in 54\% of our patients with a trend towards decreased flexion, no significant differences could be observed. We performed a post hoc power analysis and due to the small sample size, we could only acquire a power of $26 \%$. While we are limited by low power, we think a trend towards reduced flexion can be seen. Thirdly, range of motion alone is not a holistic tool for determination of functional outcome. Future studies should be performed with further clinical scores to gain a clearer understanding of postoperative function.

Lastly, the radiographic analysis has some inherent limitations as the exact position of the patient is an important presumption. For final determination of PB, we used the mISI as it has been shown to be less related to patient positioning and patella shape compared to ISI and CDI $(20,24)$.

\section{Conclusion}

Both PB and PPB are frequently observed after M-TKA with an incidence of $25 \%$ and $54 \%$, respectively. However, a reduction of $\mathrm{PB}$ and $\mathrm{PPB}$ alone does not improve postoperative range of motion.

\section{Conflicts of Interest}

All Authors declare that they have no conflicts of interest regarding this study.

\section{Authors' Contributions}

All Authors contributed in writing this manuscript. Data acquisition was performed by $\mathrm{KC}$ and $\mathrm{TG}$.

\section{Acknowledgements}

This research did not receive any specific grant from funding agencies and public, commercial, or not-for-profit sectors.

\section{References}

1 Babazadeh S, Dowsey MM, Swan JD, Stoney JD and Choong PFM: Joint line position correlates with function after primary total knee replacement: A randomised controlled trial comparing conventional and computer-assisted surgery. Bone Joint J 93: 1223-1231, 2011. PMID: 21911534. DOI: 10.1302/0301620X.93B9.26950

2 Behrend H, Graulich T, Gerlach R, Spross C and Ladurner A: Blackburne-Peel ratio predicts patients' outcomes after total knee arthroplasty. Knee Surg Sports Traumatol Arthrosc 27: 1562-1569, 2019. PMID: 29881887. DOI: 10.1007/s00167-0185016-1

3 Bellemans J: Restoring the joint line in revision TKA: Does it matter? Knee 11: 3-5, 2004. PMID: 14967319. DOI: 10.1016/S0968-0160(03)00099-1

4 Blackburne JS and Peel TE: A new method of measuring patellar height. J Bone Joint Surg Br 59: 241-242, 1977. PMID: 873986.

5 Cheuy VA, Foran JRH, Paxton RJ, Bade MJ, Zeni JA and Stevens-Lapsley JE:Arthrofibrosis associated with total knee arthroplasty. J Arthroplasty 32: 2604-2611, 2017. PMID: 28285897. DOI: $10.1016 /$ j.arth.2017.02.005

6 Chiang $\mathrm{H}$ and Jiang CC: Patella baja after revision total knee arthroplasty. Formos J Musculoskelet Disord LLC 1: 11-15, 2010. PMID: 11805927. DOI: 10.1054/arth.2002.28728

7 Chonko DJ, Lombardi AV Jr and Berend KR: Patella baja and total knee arthroplasty (TKA): etiology, diagnosis, and management. Surg Technol Int 12: 231-238, 2004. PMID: 15455331.

8 van Duijvenbode D, Stavenuiter M, Burger B, van Dijke C, Spermon $\mathrm{J}$ and Hoozemans $\mathrm{M}$. The reliability of four widely used patellar height ratios. Int Orthop 40: 493-497, 2016. PMID: 26178255. DOI: 10.1007/s00264-015-2908-2

9 Ee G, Pang HN, Chong HC, Tan MH, Lo NN and Yeo SJ: Computer navigation is a useful intra-operative tool for joint line measurement in total knee arthroplasty. Knee 20: 256-262, 2013. PMID: 23228701. DOI: 10.1016/j.knee.2012.10.026 
10 Figgie HE, Goldberg VM, Heiple KG, Moller HS and Gordon $\mathrm{NH}$ : The influence of tibial-patellofemoral location on function of the knee in patients with the posterior stabilized condylar knee prosthesis. J Bone Joint Surg Am 68: 1035-1040, 1986. PMID: 3745240.

11 Grelsamer RP Patella baja after total knee arthroplasty: Is it really patella baja? J Arthroplasty 17: 66-69, 2002. PMID: 11805927.

12 Grelsamer RP, Proctor CS and Bazos AN: Evaluation of patellar shape in the sagittal plane. Am J Sports Med 22: 61-66, 1994. PMID: 8129112. DOI: 10.1177/036354659402200111

13 Huch K, Kocak S, Kappe T, Reichel H, Jung S and Bieger R: The influence of joint line restoration on the results of revision total knee arthroplasty: comparison between distance and ratiomethods. Arch Orthop Trauma Surg 134: 537-541, 2014. PMID: 24509940. DOI: 10.1007/s00402-014-1953-4

14 Kazemi SM, Daftari Besheli L, Eajazi A, Miniator Sajadi MR, Okhovatpoor MA, Farhang Zanganeh R and Minaei R: Pseudopatella baja after total knee arthroplasty. Med Sci Monit 17: 292296, 2011. PMID: 21525812. DOI: $10.12659 / \mathrm{msm} .881770$

15 Lee J, Wang S and Kim K: Is there a difference in joint line restoration in revision Total knee arthroplasty according to prosthesis type? BMC Musculoskelet Disord 19: 1-8, 2018. PMID: 30342515. DOI: 10.1186/s12891-018-2295-0

16 Maderbacher G, Keshmiri A, Zeman F, Grifka J and Baier C: Assessing joint line positions by means of the contralateral knee: a new approach for planning knee revision surgery? Knee Surg Sport Traumatol Arthrosc 23: 3244-3250, 2015. PMID: 24996865. DOI: 10.1007/s00167-014-3157-4

17 Mason M, Belisle A, Bonutti P, Kolisek FR, Malkani A and Masini M: An accurate and reproducible method for locating the joint line during a revision total knee arthroplasty. J Arthroplasty 21: 1147-1153, 2006. PMID: 17162174. DOI: 10.1016/j.arth. 2005.08 .028
18 Pfitzner T, Perka C and Matziolis G: Patella height after total knee replacement: influence of the radiological setting. Orthopade 38 : 616-621, 2009. PMID: 19513692. DOI: 10.1007/s00132-0091437-3

19 Portner O and Pakzad H: The evaluation of patellar height: A simple method. J Bone Joint Surg Am 93: 73-80, 2011. PMID: 21209271. DOI: $10.2106 / J B J S . I .01689$

20 Rogers BA: Interobserver variation in the measurement of patellar height after total knee arthroplasty. J Bone Joint Surg Br 88: 484-488, 2006. PMID: 16567783. DOI: 10.1302/0301620X.88B4.16407

21 Scuderi GR: The stiff total knee arthroplasty: Causality and solution. J Arthroplasty 20: 23-26, 2005. PMID: 15991124. DOI: 10.1016/j.arth.2005.03.014

22 Snider MG and MacDonald SJ: The influence of the posterior cruciate ligament and component design on joint line position after primary total knee arthroplasty. J Arthroplasty 24: 10931098, 2009. PMID: 19027265. DOI: 10.1016/j.arth.2008.08.009

23 Weale AE, Orth F, Murray DW, Newman JH and Ackroyd CE: The length of the patellar tendon after unicompartmental and total knee replacement. J Bone Jt Surg (Br) 81: 790-795, 1999. PMID: 10530838. DOI: 10.1302/0301-620x.81b5.9590

24 Yang JH, Seo JG, Moon YW and Kim MH: Joint line changes after navigation-assisted mobile-bearing TKA. Orthopedics 32: 35-39, 2009. PMID: 19835306. DOI: 10.3928/0147744720090915-57 